\title{
Interview with Vladimir P Torchilin: liposomal carriers for drug delivery
}

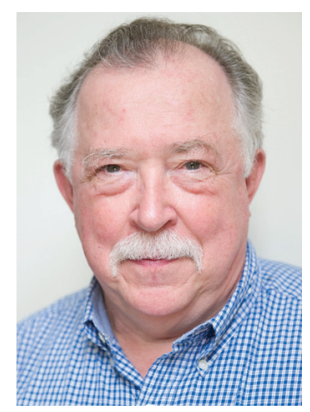

\begin{abstract}
Vladimir P Torchilin is a University Distinguished Professor and Director at the Center for Pharmaceutical Biotechnology and Nanomedicine at the School of Pharmacy, Northeastern University (MA, USA). He has published over 350 original research papers and among many other awards was the recent recipient of the 2012 Bangham Award, for his contributions to the study of liposomes. Professor Torchilin spoke to Therapeutic Delivery about the progress and challenges of the field of liposomal carriers for drug delivery as well as his own career in science to date.
\end{abstract}

Interview conducted by James Potticary, Assistant Commissioning Editor.

You have enjoyed a remarkable career spanning more than four decades. What first made you pursue a career in science?

I would name a few reasons to pursue my career specifically in chemistry. First, the science of chemistry was very popular in the USSR in the late 1950s to early 1960s, and the 'chimization of industry and agriculture' was named as a main goal by the government. This certainly had some influence. Second, I had a good chemistry teacher at high school and was impressed by the variety of very interesting and colorful transformations one can make with very simple reagents freely available at drug stores. Actually, I was caught once by my mother when I was nitrating glycerol in our kitchen. Third, many family friends of my parents were in science and I heard many of their conversations. When I began talking about my interest in becoming a historian or philosopher, they just laughed at me and explained that in addition to being interesting, science (chemistry in particular) could provide me with a 'piece of bread', which philosophy (especially with my interest to non-Marxist philosophy) would never do. This was also an important consideration.

Since the beginning of your career, how do you feel the field of targeted drug delivery has progressed?

It was pretty far from real drug targeting when I began my work with physiologically active compounds, as all targeting was derived to Ehrlich's 'magic bullet' concept. Monoclonal antibodies had not yet been suggested, and the phenomenon of using the enhanced permeability and retention (EPR) effect to target tumors was 20 years away. The 1975 paper by Helmut Ringsdorf introduced the concept of a targeted polymeric carrier, and I have to admit that we have made some quite impressive developments since then with monoclonal antibodies as therapeutic agents and ligand-targeted nanomedicines in clinical trials.

Q In light of this, what upcoming challenges do you feel are the most important to address in the next $5-10$ years?

In my opinion, the translation of bench discoveries to scaled-up products and clinical preparations will be the biggest issue. On the level of basic science, we already have a wide set of outstanding developments and very promising preparations. But, so far, only a few of those have been converted into real approved medicines. To make this happen, along with many other interesting ideas, will be the most important task.

You are perhaps most widely recognized for your research in developing liposomal carriers for cancer targeting. How much of an impact do you feel liposomes will have clinically?

First of all, there are quite a few people in the field who did as much as I have and maybe more, so I am part of that group. Liposomes are clearly the most successful of all nano-based dosage forms to date. One of the reasons for that is the fact that we have been working with liposomes as drug carriers for almost 50 years, so we have had some time. Currently, there are at least half a dozen

Vladimir P Torchilin
Department of Pharmaceutical
Sciences, Northeastern University,
Mugar Building, Room 312, 360
Huntington Avenue, Boston, MA,
021I5, USA
Tel.: +I 6173733206
Fax: +I 6173737509
E-mail: v.torchilin@neu.edu




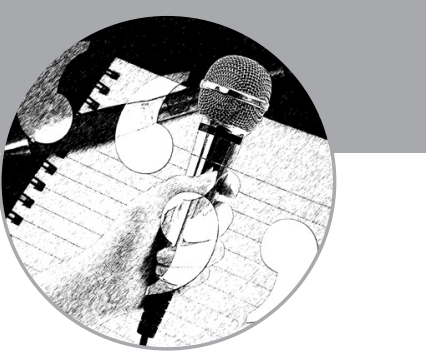

liposomal drugs approved and on the market and a few more under clinical trial. I believe that within the foreseeable future, liposomal drugs will become pretty commonplace and allow us to improve and simplify some current therapeutic approaches. However, it certainly does not exclude many other drug-delivery systems to result in other clinically useful products.

Q Liposomes within this field of therapy are currently being developed for both passive (via the EPR effect) and active targeting (e.g., antibody-targeted liposomes). Personally, do you favor one method over the other?

This all depends on the circumstances. If we need to just create a high drug concentration in a certain effected area, then EPR-based targeting could suffice. If, however, we need to deliver increased drug specifically inside cells, drug-loaded nanocarriers modified with internalizable ligands (such as antibodies) could be beneficial.

Q A major hurdle in cancer chemotherapy is multidrug resistance. Some of your recent research has demonstrated encouraging results utilizing liposomes to help overcome this. Where do you envisage these results leading?

Ideally, one would like to develop preparations that can act via more than one mechanism. For example, combining a certain means to downregulate the production of specific cell proteins involved in drug resistance (e.g., by antisense oligonucelotides or siRNA) and chemotherapeutic drugs. Clearly, you have to use a nanocarrier to have this 'double' delivery and a liposome is a very good container for this.

Q What do you see as being the best way is to improve the translation of cancer chemotherapy treatments from bench to bedside?

To have more interest from pharmaceutical and biotechnology companies. The NIH is currently trying to facilitate the interaction between basic research and industry and this is a very good sign. Despite that, this process could be both long and expensive, some recent successes with nanotechnology and drug-delivery-based approaches should attract more attention from the business world.

Other areas of your work have looked at, for example, liposomes for the diagnostics and treatment of cardiovascular disorders, and the treatment of lysosomal storage diseases. Do you think that liposomes have potential for broad therapeutic applications over a range of diseases?

Absolutely. A liposome is just a drug-delivery vehicle. You can load it with any drug, and by using very similar algorithms, send it to almost any disease site.

What has been your career highlight to date?

I have never thought about it! You always think the best is still to come, independent of your age. However, if looking seriously, it was probably the fact that one of my early works (conducted in the USSR) resulted in a real, clinically approved drug. It was a polymer-attached thrombolytic enzyme with increased stability and decreased antigenicity, which was produced and clinically utilized in the USSR for the therapy of different thrombosis from 1980 until the collapse of the USSR.

What advice would you give to aspiring scientists in the drug-delivery field? Do what you feel you have to do and be patient. Do not expect immediate success.

\section{Disclaimer}

The opinions expressed in this interview are those of the interviewee and do not necessarily reflect the views of Future Science Ltd.

\section{Financial \& competing interests disclosure}

The interviewee has no relevant affiliations or financial involvement with any organization or entity with a financial interest in or financial conflict with the subject matter or materials discussed in the manuscript. This includes employment, consultancies, honoraria, stock ownership or options, expert testimony, grants or patents received or pending, or royalties.

No writing assistance was utilized in the production of this manuscript. 